Necessary and sufficient condition for the boundedness of the Gerber-Shiu function in dependent Sparre Andersen model

\author{
Éva Orbán-Mihalykó and Csaba Mihalykó
}




\title{
NECESSARY AND SUFFICIENT CONDITION FOR THE BOUNDEDNESS OF THE GERBER-SHIU FUNCTION IN DEPENDENT SPARRE ANDERSEN MODEL
}

\author{
ÉVA ORBÁN-MIHÁLYKÓ AND CSABA MIHÁLYKÓ
}

Received 24 September, 2013

\begin{abstract}
In this paper we investigate the boundedness of the Gerber-Shiu expected discounted penalty function applied in insurance mathematics. We give a necessary and sufficient condition for its boundedness. This condition is essentially the boundedness of the conditional expectation given the ruin occurs at the first claim. It assures that the integral equation satisfied by the GerberShiu function has a unique bounded solution and it is exactly the Gerber-Shiu function. By the help of the proven necessary and sufficient condition we can also simplify the theorem concerning the necessary and sufficient condition for the limit property. We do not assume independence between the inter-claim time and the claim size but the results can be applied to the usually investigated independent case as well. We present examples to show the applicability of the condition.
\end{abstract}

2010 Mathematics Subject Classification: 91B30; 45B05; 62P05

Keywords: integral equation, Gerber-Shiu function, necessary and sufficient condition, sparre Andersen risk process

\section{INTRODUCTION}

In insurance mathematics the following stochastic process is frequently investigated ([1],[5],[7],[8])

$$
U(t)=x+c t-\sum_{k=1}^{N(t)} Y_{k} .
$$

$U(t)$ stands for the surplus of the insurance company as a function of time $t, x$ is the initial surplus of the insurance company, $c$ is the insurer's rate of the premium income per unit time, $N(t)$ is the number of claims up to time $t$ and $Y_{k}$ is the random size of the $k t h$ claim. The time periods between the consequtive claims are called interclaim times. As an explanation, $c t$ stands for the income of the company to $t$, while $\sum_{k=1}^{N(t)} Y_{k}$ is its expenses to $t$ paid for the insurers. The process $U(t)$ is briefly called as Sparre Andersen risk process.

In risk theory the main topics of interest are the probability of ruin, the moments of the time of ruin, the distribution of the surplus before ruin and the deficit at ruin. 
Ruin means that the surplus falls below zero, time of ruin is the time point when it first happens. To answer these questions, the Gerber-Shiu expected discounted penalty function was introduced by Gerber and Shiu in [6],[7] and was investigated in lots of papers (for example [1],[5],[8],[10]).

In the papers dealing with determination of the Gerber-Shiu function, usually an integral equation is set up for it and solved with a boundary condition (e.g.[1], [8]). Researchers use the boundary condition to select the Gerber-Shiu function from the solutions of the integral equation. In [1],[8], the authors use the boundary condition that the expected discounted penalty function tends to zero if its argument tends to infinity. This implies the boundedness of the function investigated, as well. However, the mentioned limit property does not always hold, moreover, the Gerber-Shiu function may be unbounded as well. It strictly depends on the penalty funtion. Examples for these cases are presented in [9]. The identification of the Gerber-Shiu function in the set of solutions is rather connected to its boundedness than to its limit. In this paper we present such a condition (condition A in Section 2) which assures the boundedness of the Gerber-Shiu function. This condition for the boundedness of the Gerber-Shiu function coincides with the condition for uniqueness of the bounded solution of the integral equation. It concerns the penalty function and the joint density function of inter-claim times and claim sizes but it is not a condition on the solution itself.

Providing a necessary and sufficient condition for the limit property in [9], boundedness of the Gerber-Shiu function was assumed. In applications it was checked in each case by different methods based on estimations. Now condition A makes easier to check the boundedness.

Researchers usually assume independence between the inter-claim times and claim size but recently some results have been published for models that allow for dependence between claim size and claim-time ([2],[3],[4],[5],[11]). In this paper we examine the generalized case.

Let us consider the risk model with the following notations: $T_{0}=0, T_{k}, k=$ $1,2,3, \ldots$ are the inter-arrival times, $Y_{k}$ is the $k t h$ claim size, $\left(T_{k}, Y_{k}\right)$ has joint distribution function $H(t, y)$ and density function $h(t, y)$ for any value of $k$. We suppose that $\left(T_{k}, Y_{k}\right)$ are independent for $k=1,2,3, \ldots$ but the random variables $T_{k}$ and $Y_{k}$ may be dependent for fixed values of $k=1,2,3 \ldots$ The marginal distribution function of $T_{k}$ is denoted by $F(t)$, its density function by $f(t)$, where $T_{k}$ is nonnegative with finite expectation $\mu_{f}$. The marginal distribution function of $Y_{k}$ is denoted by $G(y)$, its density function by $g(y)$, where $Y_{k}$ is nonnegative with finite expectation $\mu_{g}$. The number of claims up to time $t$ is given by

$$
N(t)= \begin{cases}0, & \text { if } t \leq T_{1} \\ n, & \text { if } \sum_{k=1}^{n} T_{k}<t \text { and } t \leq \sum_{k=1}^{n+1} T_{k} .\end{cases}
$$

The ultimate probability of ruin is 


$$
\Psi(x)=P\left(x+c t-\sum_{k=1}^{N(t)} Y_{k}<0 \text { for some } 0 \leq t\right) .
$$

The time of ruin is

$$
T= \begin{cases}\inf \{t: U(t)<0\}, & \text { if there exists } 0 \leq t, \text { such that } U(t)<0, \\ \infty, & \text { if } 0 \leq U(t) \text { for all } 0 \leq t .\end{cases}
$$

Let $w: \mathbb{R}_{0}^{+} \mathbf{x} \mathbb{R}_{0}^{+} \rightarrow \mathbb{R}_{0}^{+}$be the penalty function.

The Gerber-Shiu expected discounted penalty function is defined as

$$
m_{\delta}(x)=\mathbb{E}\left(e^{-\delta T} \cdot w\left(U\left(T^{-}\right),|U(T)|\right) \cdot 1_{T<\infty} \mid U(0)=x\right),
$$

if the expectation is finite. Here $0 \leq x, 0 \leq \delta$ and $U\left(T^{-}\right)$is the surplus immediately before ruin, $|U(T)|$ is the deficit at ruin. Note that $0 \leq m_{\delta}(x)$.

Applying appropriate penalty functions, the Gerber-Shiu function is suitable for investigating some important characteristics of the process. For example if we use penalty function $w(x, y) \equiv 1$ and substitute $\delta=0$ we get

$$
m_{0}(x)=\mathbb{E}\left(1_{T<\infty} \mid U(0)=x\right)=\Psi(x),
$$

furthermore $\left.\frac{\partial m_{\delta}(x)}{\partial \delta}\right|_{\delta=0}=-\mathbb{E}\left(1_{T<\infty}\right)$. If we apply penalty function $w(x, y)=x^{n}$ and substitute $\delta=0, m_{0}(x)=\mathbb{E}\left(U\left(T^{-}\right)^{n} \cdot 1_{T<\infty} \mid U(0)=x\right)$, which is the $n$th moments of the surplus before ruin. If $w(x, y)=y$ then

$$
m_{0}(x)=\mathbb{E}\left(|U(T)| \cdot 1_{T<\infty} \mid U(0)=x\right)
$$

is the expectation of deficit at ruin.

For the sake of simplicity in this paper we suppose that $h$ and $w$ are continuous functions on $[0, \infty) \mathrm{x}[0, \infty)$ and so is $m_{\delta}(x)$. We note that $U(0)=x$ follows from the definition of $U(t)$, hence in the following we do not indicate it.

In the next section of the paper we prove a necessary and sufficient condition for the boundedness of $m_{\delta}(x)$ and we present some examples to show the applicability of the theorem. Then we conclude the consequences of the theorem concerning the integral equation satisfied by the Gerber-Shiu function and we present a necessary and sufficient condition for the limit property $\lim _{x \rightarrow \infty} m_{\delta}(x)=0$.

\section{A NECESSARY AND SUFFICIENT CONDITION FOR THE BOUNDEDNESS}

Theorem 1. Suppose

$$
S(x)=\int_{0}^{\infty} \int_{x+c t}^{\infty} e^{-\delta t} w(x+c t, y-c t-x) h(t, y) d y d t<\infty
$$

for all $0 \leq x$ and let $0<\delta$ be fixed.

Then, the Gerber-Shiu function $m_{\delta}(x)$ is bounded if and only if

$$
\sup _{0 \leq x} S(x)=\sup _{0 \leq x} \int_{0}^{\infty} \int_{x+c t}^{\infty} e^{-\delta t} w(x+c t, y-c t-x) h(t, y) d y d t<\infty .
$$


Proof. First we suppose that $m_{\delta}(x)$ is a bounded function of $x$, and we prove that

$$
\kappa=\sup _{0 \leq x} S(x)<\infty .
$$

The non-negativity of the functions $h$ and $w$ implies the non-negativity of $S$ as well. Let $A_{i}$ be the event that the ruin occurs at the $i t h$ claim, $i=1,2,3, \ldots$. Notice that

$$
1_{T<\infty}=\sum_{i=1}^{\infty} 1_{A_{i}} .
$$

Now

$$
\begin{aligned}
m_{\delta}(x)=\mathbb{E}\left(e^{-\delta T} \cdot w\right. & \left.\left(U\left(T^{-}\right),|U(T)|\right) \cdot 1_{T<\infty}\right) \geq \\
& \mathbb{E}\left(e^{-\delta T} \cdot w\left(U\left(T^{-}\right),|U(T)|\right) \cdot 1_{A_{1}}\right)= \\
= & \int_{0}^{\infty} \int_{x+c t}^{\infty} e^{-\delta t} w(x+c t, y-c t-x) h(t, y) d y d t=S(x) .
\end{aligned}
$$

Therefore, if $m_{\delta}(x) \leq K$, then $S(x) \leq K$, consequently $\kappa=\sup _{0 \leq x} S(x) \leq K<\infty$.

Conversely, we suppose that $\kappa=\sup _{0 \leq x} S(x)<\infty$. We prove that in this case $m_{\delta}(x)$ is bounded.

Now

$$
\begin{aligned}
m_{\delta}(x) & =\mathbb{E}\left(e^{-\delta T} \cdot w\left(U\left(T^{-}\right),|U(T)|\right) \cdot\left(\sum_{i=1}^{\infty} 1_{A_{i}}\right)\right)= \\
& =\sum_{i=1}^{\infty} \mathbb{E}\left(e^{-\delta T} \cdot w\left(U\left(T^{-}\right),|U(T)|\right) \cdot 1_{A_{i}}\right) .
\end{aligned}
$$

Let

$$
m_{\delta}\left(x ; A_{i}\right)=\mathbb{E}\left(e^{-\delta T} \cdot w\left(U\left(T^{-}\right),|U(T)|\right) \cdot 1_{A_{i}}\right), \quad i=1,2,3, \ldots
$$

Then

$$
m_{\delta}\left(x ; A_{1}\right)=\int_{0}^{\infty} \int_{x+c t}^{\infty} e^{-\delta t} w(x+c t, y-c t-x) h(t, y) d y d t=S(x)<\infty
$$

according to condition $(A)$.

If $A_{2}$ holds, then

$$
\begin{gathered}
T=T_{1}+T_{2}, \quad U\left(T^{-}\right)=x+c T_{1}-Y_{1}+c T_{2}, \\
U(T)=x+c T_{1}-Y_{1}+c T_{2}-Y_{2}
\end{gathered}
$$


and

$$
\begin{aligned}
m_{\delta}\left(x ; A_{2}\right) & =\int_{0}^{\infty} \int_{0}^{x+c t_{1}} e^{-\delta t_{1}} . \\
& \int_{0}^{\infty} \int_{x+c t_{1}-y_{1}+c t_{2}}^{\infty} e^{-\delta t_{2} .} \\
& w\left(x+c t_{1}-y_{1}+c t_{2}, y_{2}-\left(x+c t_{1}-y_{1}+c t_{2}\right)\right) . \\
& \cdot h\left(t_{2}, y_{2}\right) d y_{2} d t_{2} \cdot h\left(t_{1}, y_{1}\right) d y_{1} d t_{1}
\end{aligned}
$$

If we use the notation $z=x+c t_{1}-y_{1}$, then $0 \leq z$, as $A_{1}$ does not hold. The inner integral of the previous formula has the form

$$
\begin{aligned}
& \int_{0}^{\infty} \int_{x+c t_{1}-y_{1}+c t_{2}}^{\infty} e^{-\delta t_{2}} w\left(x+c t_{1}-y_{1}+c t_{2}, y_{2}-\left(x+c t_{1}-y_{1}+c t_{2}\right)\right) h\left(t_{2}, y_{2}\right) d y_{2} d t_{2} \\
& =\int_{0}^{\infty} \int_{z+c t_{2}}^{\infty} e^{-\delta t_{2}} w\left(z+c t_{2}, y_{2}-\left(z+c t_{2}\right)\right) h\left(t_{2}, y_{2}\right) d y_{2} d t_{2}=m_{\delta}\left(x+c t_{1}-y_{1} ; A_{1}\right) .
\end{aligned}
$$

Now we can use the following estimate:

$$
\begin{aligned}
m_{\delta}\left(x ; A_{2}\right) & =\int_{0}^{\infty} \int_{0}^{x+c t_{1}} e^{-\delta t_{1}} m_{\delta}\left(x+c t_{1}-y_{1} ; A_{1}\right) h\left(t_{1}, y_{1}\right) d y_{1} d t_{1} \\
& \leq \int_{0}^{\infty} \int_{0}^{\infty} e^{-\delta t_{1}} \cdot \kappa \cdot h\left(t_{1}, y_{1}\right) d y_{1} d t_{1}=\kappa \int_{0}^{\infty} e^{-\delta t_{1}} f\left(t_{1}\right) d t_{1}=\kappa \hat{f}(\delta),
\end{aligned}
$$

where $\hat{f}(\delta)$ is the Laplace transform of the density function $f$. Note that if $0<\delta$, then $\hat{f}(\delta)<1$.

In general, for $2 \leq i$, the following recursion holds:

$$
m_{\delta}\left(x ; A_{i}\right)=\int_{0}^{\infty} \int_{0}^{x+c t_{1}} e^{-\delta t_{1}} m_{\delta}\left(x+c t_{1}-y_{1} ; A_{i-1}\right) h\left(t_{1}, y_{1}\right) d y_{1} d t_{1} .
$$

This formula can be proved with the following argument: take the conditional expectation on the first claim time and size. If $A_{i}$ holds for some $2 \leq i$, then the ruin does not occur at the first claim, consequently $Y_{1} \leq x+c T_{1}$. After the first claim, the "initial" surplus is $x+c T_{1}-Y_{1}$, the number of claims to ruin is decreased. If $T_{1}=t_{1}$ and $Y_{1}=y_{1}$, then the expectation of the function $e^{-\delta T} \cdot w\left(U\left(T^{-}\right),|U(T)|\right)$ from this time point $t_{1}$ and with initial surplus $x+c t_{1}-y_{1}$ is $m_{\delta}\left(x+c t_{1}-y_{1} ; A_{i-1}\right)$. The time to ruin from zero is $t_{1}$ more than the time to ruin from the time $T_{1}$, which is the 
reason for multiplication by $e^{-\delta t_{1}}$. Consequently,

$$
\begin{aligned}
m_{\delta}\left(x ; A_{i}\right) & =\mathbb{E}\left(e^{-\delta T} w\left(U\left(T^{-}\right),|U(T)|\right) \cdot 1_{A_{i}}\right)= \\
& =\mathbb{E}\left(\mathbb{E}\left(e^{-\delta T} w\left(U\left(T^{-}\right),|U(T)|\right) \cdot 1_{A_{i}} \mid T_{1}, Y_{1}\right)\right)= \\
& =\int_{0}^{\infty} \int_{0}^{x+c t_{1}} e^{-\delta t_{1}} m_{\delta}\left(x+c t_{1}-y_{1} ; A_{i-1}\right) h\left(t_{1}, y_{1}\right) d y_{1} d t_{1}
\end{aligned}
$$

Now if

$$
m_{\delta}\left(x+c t_{1}-y_{1} ; A_{i-1}\right) \leq K_{i-1},
$$

then

$$
m_{\delta}\left(x ; A_{i}\right) \leq \int_{0}^{\infty} \int_{0}^{\infty} e^{-\delta t_{1}} m_{\delta}\left(x+c t_{1}-y_{1} ; A_{i-1}\right) h\left(t_{1}, y_{1}\right) d y_{1} d t_{1} \leq K_{i-1} \hat{f}(\delta)
$$

We have seen that

$$
m_{\delta}\left(x ; A_{2}\right) \leq \kappa \hat{f}(\delta)
$$

hence

$$
m_{\delta}\left(x ; A_{i}\right) \leq \kappa(\hat{f}(\delta))^{i-1}, i \geq 3
$$

also holds, consequently the inequality

$$
m_{\delta}(x) \leq \sum_{i=1}^{\infty} \kappa(\hat{f}(\delta))^{i-1}=\frac{\kappa}{1-\hat{f}(\delta)}
$$

is satisfied, which implies the boundedness of $m_{\delta}(x)$.

Remark 1 . Notice that the necessary and sufficient condition for the boundedness of is $m_{\delta}(x)$ essentially the boundedness of the conditional expectation given the ruin occurs at the first claim.

Remark 2. In insurance mathematics the net profit condition $\left(0<c \mu_{f}-\mu_{g}\right)$ is usually assumed. Net profit condition expresses that the income per unit time is more than the average expenses per unit time. Condition A can be satisfied even in the case when the usual net profit condition does not hold. Consequently, the net profit condition is not necessary for the boundedness of the Gerber-Shiu function if $\delta>0$.

Let us investigate some examples when the boundedness of $m_{\delta}(x)$ holds and when it does not hold. In Examples 1,2,3 we suppose that

$$
h(t, y)=f(t) g(y),
$$

that is we assume the independence between the inter-claim times and claim sizes. In Example 4 we deal with a dependent case. 
Example 1. Let

$$
w(x, y)=x^{n}, 1 \leq n
$$

If

$$
\lim _{y \rightarrow \infty} y^{n}(1-G(y))
$$

is finite, then $m_{\delta}(x)$ is bounded.

Let us investigate $S(x)$ and prove its boundedness.

$$
\begin{aligned}
S(x) & =\int_{0}^{\infty} \int_{x+c t}^{\infty} e^{-\delta t} w(x+c t, y-c t-x) h(t, y) d y d t= \\
& =\int_{0}^{\infty} \int_{x+c t}^{\infty} e^{-\delta t}(x+c t)^{n} f(t) g(y) d y d t= \\
& =\int_{0}^{\infty} e^{-\delta t}(x+c t)^{n} f(t)\left(\int_{x+c t}^{\infty} g(y) d y\right) d t= \\
& =\int_{0}^{\infty} e^{-\delta t}(x+c t)^{n} f(t)(1-G(x+c t)) d t \leq \\
& \leq K \int_{0}^{\infty} e^{-\delta t} f(t) d t
\end{aligned}
$$

as

$$
\lim _{y \rightarrow \infty} y^{n}(1-G(y)) \leq K_{1}
$$

implies

$$
(x+c t)^{n}(1-G(x+c t)) \leq K
$$

for appropriate value of $K$.

Consequently $\sup S(x)$ is finite, hence applying Theorem 1 we can conclude that $m_{\delta}(x)$ is bounded.

We note that if the $n t h$ moment of $Y_{k}$ is finite, then $\lim _{y \rightarrow \infty} y^{n}(1-G(y))=0$. Hence in that case the Gerber-Shiu function is bounded.

Example 2. Let

$$
w(x, y)=y .
$$

If

$$
\lim _{y \rightarrow \infty} y(1-G(y))
$$

is finite, then $m_{\delta}(x)$ is bounded.

Let us investigate $S(x)$ again and prove its boundedness.

$$
S(x)=\int_{0}^{\infty} \int_{x+c t}^{\infty} e^{-\delta t} w(x+c t, y-c t-x) h(t, y) d y d t=
$$




$$
\begin{aligned}
& =\int_{0}^{\infty} \int_{x+c t}^{\infty} e^{-\delta t}(y-c t-x) f(t) g(y) d y d t= \\
& =\int_{0}^{\infty} e^{-\delta t} f(t)\left(\int_{x+c t}^{\infty}(y-c t-x) g(y) d y\right) d t \leq \\
& \leq \int_{0}^{\infty} e^{-\delta t} f(t)\left(\mu_{g}-(x+c t)(1-G(x+c t))\right) d t \leq \\
& \leq K \int_{0}^{\infty} e^{-\delta t} f(t) d t=K \cdot \hat{f}(\delta)
\end{aligned}
$$

as $\lim _{y \rightarrow \infty} y(1-G(y)) \leq K_{1}$ implies the inequality

$$
0 \leq \mu_{g}-(x+c t)(1-G(x+c t)) \leq K
$$

for appropriate value of $K$.

We point out that in both cases the tails of the distribution function of the claim sizes determine the boundedness of the Gerber-Shiu function. So does in our next example as well.

\section{Example 3. Let}

$$
w(x, y)=x^{3}
$$

and

$$
h(t, y)=f(t) \cdot \frac{2}{(1+y)^{3}}, \quad y \geq 0,
$$

and let $f(t)$ be an arbitrary continuous density function. In this case $m_{\delta}(x)$ is not bounded.

Let us investigate $S(x)$ again and prove that condition A does not hold.

$$
\begin{aligned}
S(x) & =\int_{0}^{\infty} \int_{c t+x}^{\infty} e^{-\delta t}(x+c t)^{3} f(t) g(y) d y d t= \\
& =\int_{0}^{\infty} e^{-\delta t}(x+c t)^{3} f(t) \frac{1}{(1+c t+x)^{2}} d t \geq \\
& \geq \int_{0}^{\infty} e^{-\delta t} f(t)(x+c t-2) d t \geq \\
& \geq(x-2) \int_{0}^{\infty} e^{-\delta t} f(t) d t=(x-2) \hat{f}(\delta),
\end{aligned}
$$

consequently

$$
\sup _{0 \leq x} S(x)=\infty
$$

Applying Theorem 1 we can see that $m_{\delta}(x)$ is not bounded. 
Similar arguments can be given for other heavy tailed distributions of claim sizes with penalty function

$$
w(x, y)=x^{n}
$$

with appropriate large exponent $n$.

Finally, we present an example which shows the applicability of Theorem 1 in the case of dependence between inter-claim times and claim sizes.

Example 4. Let

$$
w(x, y)=x^{n}
$$

and

$$
H(t, y)=F_{1}(t)\left(e^{-\beta t} G_{1}(y)+\left(1-e^{-\beta t}\right) G_{2}(y)\right)
$$

(mentioned in [5]), where $F_{1}(t), G_{1}(y), G_{2}(y)$ are distribution functions with density functions $f_{1}(t), g_{1}(y), g_{2}(y)$ respectively. If $G_{1}(y)$ and $G_{2}(y)$ have finite nth moments, then the function $m_{\delta}(x)$ is bounded. This can be seen as follows: first we note that finite nth moments imply

$$
\lim _{y \rightarrow \infty} y^{n}\left(1-G_{1}(y)\right)=0
$$

and

$$
\lim _{y \rightarrow \infty} y^{n}\left(1-G_{2}(y)\right)=0,
$$

hence

$$
0 \leq y^{n}\left(1-G_{1}(y)\right) \leq K_{1}
$$

and

$$
0 \leq y^{n}\left(1-G_{2}(y)\right) \leq K_{2}
$$

hold with appropriate constant values. Moreover,

$$
\begin{gathered}
h(t, y)=\frac{\partial^{2} H(t, y)}{\partial t \partial y}=f_{1}(t)\left(e^{-\beta t} g_{1}(y)+\left(1-e^{-\beta t}\right) g_{2}(y)\right)+ \\
F_{1}(t)\left(-\beta e^{-\beta t} g_{1}(y)+\beta e^{-\beta t} g_{2}(y)\right),
\end{gathered}
$$

hence

$$
h(t, y) \leq\left(f_{1}(t)+\beta e^{-\beta t}\right)\left(g_{1}(y)+g_{2}(y)\right) .
$$

We can see that

$$
\sup _{0 \leq x} S(x)
$$

is finite, as

$$
\begin{aligned}
S(x) & =\int_{0}^{\infty} \int_{c t+x}^{\infty} e^{-\delta t}(x+c t)^{n} h(t, y) d y d t \\
& \leq \int_{0}^{\infty} e^{-\delta t}(x+c t)^{n}\left(f_{1}(t)+\beta e^{-\beta t}\right)\left(\int_{c t+x}^{\infty} g_{1}(y)+g_{2}(y) d y\right) d t
\end{aligned}
$$




$$
\begin{aligned}
& =\int_{0}^{\infty} e^{-\delta t}(x+c t)^{n}\left(f_{1}(t)+\beta e^{-\beta t}\right)\left(1-G_{1}(x+c t)+1-G_{2}(x+c t)\right) d t \\
& \leq \int_{0}^{\infty} e^{-\delta t}\left(f_{1}(t)+\beta e^{-\beta t}\right)\left(K_{1}+K_{2}\right) d t \\
& \leq\left(K_{1}+K_{2}\right)\left(\hat{f}_{1}(\delta)+\frac{\beta}{\beta+\delta}\right) .
\end{aligned}
$$

Applying Theorem 1 we can conclude that $m_{\delta}(x)$ is bounded.

We note that examples similar to Example 2 and Example 3 can be constructed applying appropriate dependent joint density function in the case of penalty functions $w(x, y)=y$ and $w(x, y)=x^{3}$.

Remark 3. In [9], in Theorem 2 the authors prove that if condition (A) holds, then the integral equation satisfied by $m_{\delta}(x)$ (Eq. (3) in that paper, which is a generalization of the Eq.(1) in [1]),

$$
\begin{aligned}
\Phi_{\delta}(x) & =\int_{0}^{\infty} \int_{0}^{x+c t} e^{-\delta t_{1}} \Phi_{\delta}(x+c t-y) \cdot h(t, y) d y d t+ \\
& +\int_{0}^{\infty} \int_{x+c t}^{\infty} e^{-\delta t} \cdot w(x+c t, y-(x+c t)) d y d t
\end{aligned}
$$

has a unique solution in the set of bounded functions. Now Theorem 1 states that if condition (A) holds, then $m_{\delta}(x)$ is bounded. Consequently if condition (A) holds, then the unique bounded solution of the above integral equation equals $m_{\delta}(x)$. If condition (A) does not hold, the bounded solution of the equation (if it exists) is not the Gerber-Shiu function. Consequently, condition (A) is suitable for selecting the function $m_{\delta}(x)$ from the bounded and unbounded solutions of the integral equation satisfied by the Gerber-Shiu function.

Finally, we use Theorem 1 to simplify the theorem giving a necessary and sufficient condition for the limit property $\lim _{x \rightarrow \infty} m_{\delta}(x)=0$. In this version we do not have any assumption concerning boundedness of $m_{\delta}(x)$.

First we recall Theorem 3 from [9].

Proposition 1. Let $0<\delta$ be fixed and suppose that $m_{\delta}(x)$ is bounded. Then $\lim _{x \rightarrow \infty} m_{\delta}(x)=0$ if and only if

$$
\limsup _{0 \leq x} S(x)=0 .
$$


Theorem 2. Let $S(x)$ be finite for all $0 \leq x$ and let $0<\delta$ be fixed. Then

$$
\lim _{x \rightarrow \infty} m_{\delta}(x)=0
$$

if and only if

$$
\limsup _{0 \leq x} S(x)=0 .
$$

Proof. It is obvious that condition $(B)$ implies condition $(A)$. Consequently, by Theorem 1, we can conclude that $m_{\delta}(x)$ is bounded if condition B holds. Taking the previous Proposition into account, we can state that $\lim _{x \rightarrow \infty} m_{\delta}(x)=0$ holds as well.

Conversely, $\lim _{x \rightarrow \infty} m_{\delta}(x)=0$ implies boundedness of the function $m_{\delta}(x)$, as $m_{\delta}(x)$ is continuous. Recalling the previous Proposition again, we can conclude that $\lim \sup S(x)=0$.

$0 \leq x$

\section{CONCLUSION}

In this paper a necessary and sufficient condition was proved for the boundedness of the frequently used Gerber-Shiu function. This condition is essentially the boundedness of the conditional expectation given the ruin occurs at the first claim. It assures that the integral equation satisfied by the Gerber-Shiu function (Eq.2.1) has a unique bounded solution and it is exactly the Gerber-Shiu function. The condition implies that the bounded property of the Gerber-Shiu function is the consequence of the choice of the penalty function and the density function, moreover it can be checked in the knowledge of the penalty function and the joint density function.

\section{REFERENCES}

[1] H. Albrecher, C. Constantinescu, G. Pirsic, G. Regensburger, and M. Rosenkranz, "An algebraic operator approach to the analysis of Gerber-Shiu functions," Insur. Math. Econ., vol. 46, no. 1, pp. 42-51, 2010

[2] H. Albrecher and J. L. Teugels, "Exponential behavior in the presence of dependence in risk theory," J. Appl. Probab., vol. 43, no. 1, pp. 257-273, 2006.

[3] R. S. Ambagaspitiya, "Ultimate ruin probability in the Sparre Andersen model with dependent claim sizes and claim occurrence times," Insur. Math. Econ., vol. 44, no. 3, pp. 464-472, 2009.

[4] A. L. Badescu, E. C. Cheung, and D. Landriault, "Dependent risk models with bivariate phase-type distributions," J. Appl. Probab., vol. 46, no. 1, pp. 113-131, 2009.

[5] E. C. Cheung, D. Landriault, G. E. Willmot, and J.-K. Woo, "Structural properties of Gerber-Shiu functions in dependent Sparre Andersen models," Insur. Math. Econ., vol. 46, no. 1, pp. 117-126, 2010.

[6] H. U. Gerber and E. S. Shiu, "The joint distribution of the time of ruin, the surplus immediately before ruin, and the deficit at ruin," Insur. Math. Econ., vol. 21, no. 2, pp. 129-137, 1997.

[7] H. U. Gerber and E. S. Shiu, "On the time value of ruin. With discussion and a reply by the authors," N. Am. Actuar. J., vol. 2, no. 1, pp. 48-78, 1998.

[8] H. U. Gerber and E. S. Shiu, "The time value of ruin in a Sparre Andersen model. With discussion and a reply by the authors," N. Am. Actuar. J., vol. 9, no. 2, pp. 49-84, 2005. 
[9] E. O. Mihálykó and C. Mihálykó, "Mathematical investigation of the Gerber-Shiu function in the case of dependent inter-claim time and claim size," Insur. Math. Econ., vol. 48, no. 3, pp. 378-383, 2011.

[10] G. E. Willmot, "On the discounted penalty function in the renewal risk model with general interclaim times," Insur. Math. Econ., vol. 41, no. 1, pp. 17-31, 2007.

[11] Z. Zhang and H. Yang, "Gerber-Shiu analysis in a perturbed risk model with dependence between claim sizes and interclaim times," J. Comput. Appl. Math., vol. 235, no. 5, pp. 1189-1204, 2011.

Authors' addresses

Éva Orbán-Mihálykó

University of Pannonia, Department of Mathematics, 10 Egyetem St., H-8200 Veszprém, Hungary

E-mail address: orbane@almos. uni-pannon. hu

Csaba Mihálykó

University of Pannonia, Department of Mathematics, 10 Egyetem St., H-8200 Veszprém, Hungary

E-mail address: mihalykodalmos. uni-pannon. hu 